\title{
Energy Coin: A Universal Digital Currency Based on Free Energy
}

\author{
Kang Jian \\ Wanzs Energy Science Inc. Beijing, China \\ Email address: \\ kangiianbj@163.com \\ To cite this article: \\ Kang Jian. Energy Coin: A Universal Digital Currency Based on Free Energy. American Journal of Modern Energy. \\ Vol. 6, No. 5, 2020, pp. 95-100. doi: 10.11648/j.ajme.20200605.11
}

Received: November 17, 2020; Accepted: December 1, 2020; Published: December 8, 2020

\begin{abstract}
This paper introduces a kind of world common digital currency based on free energy, which scientifically defines value and wealth, consummates the theoretical basis of economics, and points out that energy coin is an ideal world digital common currency for wealth and freedom. free energy $(\mathrm{G}=\mathrm{H}-\mathrm{TS})$ of the earth's ecosystem is the dynamical potential of its self-organization and evolution and the state function that dominates the direction of evolution. Human society is an integral part of the earth's ecosystem. In social and economic activities, free energy (G=H-TS) is expressed as the value of goods and services, and is the potential function that dominates social progress. The energy coin, which uses free energy as the benchmark of value measurement, fully reflects the nature of value ecology, politics, economics and physics. The essence of energy coin is thermodynamic potential function, driving force of human society, and benefit mechanism of system self-organization and cooperation. Free energy is the thermodynamic potential of goods and services, representing the ability of external work and empowerment. Energy coins are physically decentralized based on the energy contained in countless goods and services, and free energy is measurable and calculated, so energy coins are a natural non-national ideal world currency, that is not manipulated by any individual, organization or state, and unifies the self-organization mechanism of the earth's ecosystem with the market self-organization mechanism. Energy coin is a value chain digital currency based on block chain technology. It is a monetary system that can automatically control the size of money according to the scale of goods and services market. It solves the pain points of various currencies in history, such as gold deflation, inflation of legal currency, and the malpractice of no value base of various digital currency.
\end{abstract}

Keywords: Free Energy, Value Measurement, Digital Currency, Block Chain, Value Chain, Energy Coin

\section{Introduction}

The energy in nature is infinite, but the energy that human beings can use is limited and scarce, and the energy that is useful to human beings is the free energy that can be transformed into the earth's ecosystem and social system. The essence of all the history of human civilization is the history of constantly obtaining the energy of survival and development. It is found that the physical nature of economic wealth and value is free energy, which is the thermodynamic potential of goods and services, representing an ability to do external work and empower, it also represents the increase in the expected value and wealth of goods and services to customers.

This paper introduces an energy coin based on free energy, It is the ideal currency to describe the essence of the value chain of earth civilization and the motive force of development. Free energy of the earth's ecosystem is the dynamical potential [1] of its self-organization and evolution and the state function that dominates the direction of evolution. Human society is an integral part of the earth's ecosystem. In social and economic activities, free energy is the value of goods and services, and it is the potential function that dominates social progress. The energy coin with free energy as the benchmark of value reflects the essence of ecology, politics, economics and physics of value. The essence of energy coin is thermodynamic potential function, driving force of human society and benefit mechanism of ecosystem self-organization and cooperative cooperation.

The value chain of energy coins can cover all goods and services horizontally and vertically back to the source of all values, as shown in Table 1. The vertical order of value chain and currency form of energy coin is: atomic energy $\rightarrow$ 
ecosystem free energy $\rightarrow$ social system free energy $\rightarrow$ general equivalent currency $\rightarrow$ credit currency $\rightarrow$ legal

currency $\rightarrow$ digital currency $\rightarrow$ bitcoin $\rightarrow$ energy coin.

Table 1. Traceability of Energy Coin Value Chain.

\begin{tabular}{lll}
\hline Circle and morphology & Association characteristics & Vertical order of value chains and currency patterns \\
\hline Universe, Solar System, Earth & Solar energy, Earth's inner energy & Atomic energy: nuclear fusion, nuclear fission \\
Earth ecosystem & Life, photosynthesis, microorganisms, animals and plants & Ecosystem free energy: molecular energy, wind energy, \\
& Natural persons & water energy, phase change energy \\
Human society & Race, tribe, country, economy & Free energy of the social system: goods, values, wealth, \\
& general equivalent money \\
Organization & Authoritarianism, centralization & Credit currency, legal tender \\
Internet & Government, Enterprises, social groups & Digital currency \\
Block chain & Platform, Networking, De-leveling & Bitcoin, \\
\hline
\end{tabular}

Table 1 shows, that the source of all values comes from the atomic energy of the solar system and the earth. The earth's ecosystem converts solar energy into chemical energy, wind energy, water energy, biological energy, electric energy and other valuable free energy for human beings through processes such as photosynthesis and human social production. So the source of value is energy.

\section{Theory of Dissipative Structure, Free Energy State Function and Brief Introduction of Earth Ecosystem}

A theory of dissipative structure was proposed by Prigogine in 1969 , which is used to describe the common dynamic characteristics of non-equilibrium thermodynamic systems such as life and ecosystem away from equilibrium [12-14]. The school represented by Hakone of Germany and Suzuki of Japan has made important contributions to the establishment and development of dissipative structure theory away from equilibrium state. However, the theory is still in the primary stage, and new concepts, laws and mathematical tools [6] need to be explored. we find that the free energy state function can be used as a mathematical tool to generally describe the state, order, structure and dynamics characteristics of various dissipative structures.

In order to understand the essential difference between dissipative structure and balanced structure, examples are given to illustrate that beautiful women are like dissipative structures, which need the energy of food to maintain their appearance and body, while beds are balanced structures. It does not need energy to maintain its structure and shape. As large as the earth's ecosystem, human society, as small as people, animals and plants, microbes and other living systems are dissipative structures, all need to maintain their state, order and structure by constantly consuming energy. Without sufficient energy flow density, the system will shrink and collapse.

The earth ecosystem is a dissipative structure driven by solar energy (nuclear fusion) and the earth's inner energy (nuclear fission). human society is a component of the earth's ecosystem, and its state, order, structure and motion law can be quantitatively described by the free energy state function $[7,18]$. The earth's ecosystem, human society and life are all dissipative structures that need energy to maintain their state, order and structure, and energy is the driving force for the survival, development and prosperity of the earth's civilization.

The purpose of all human economic activities is to obtain the energy of survival and development from nature. Therefore, the definition of value in economics by energy reveals the foundation of earth civilization, the ethical relationship between man and nature, and the essence of value and wealth in economics. Free energy is the thermodynamic potential of goods and services. It represents a kind of ability to do work and enable external work. It defines "the value of economics" by "free energy of physics ", which not only scientifically reflects the physical essence of value. It also expresses the social and natural ecological attributes of value in economics.

\section{Use Free Energy to Define Wealth and Value}

It must be pointed out that from the classical labor value theory put forward by Adam Smith [8], Karl Heinrich Marx and John Maynard Keynes [10-11] to the labor value theory of modern economics, the cost of goods and services has been wrongly regarded as value. This error is the product of the process of human exploration of truth, the historical limitation of epistemology.

In fact, the value essence of goods and services is free energy [2,3]. Value $\Delta \mathrm{G}$ is the increase in the expected value of goods and services to customers $\Delta \mathrm{H}$ less the $\mathrm{T} \Delta \mathrm{S}$ of the cost of purchasing or producing goods and services. Value is the potential growth of goods and services to customers, such as expected material wealth, quality of life, work efficiency, social consensus, knowledge and cognition.

It can be represented by formula (1):

$$
\Delta \mathrm{G}=\Delta \mathrm{H}-\mathrm{T} \Delta \mathrm{S}
$$

The value of goods or services is usually realized by the buyer and seller through the price in the exchange process. The value of the goods for the buyer is $\Delta \mathrm{H}$ minus the price; for the seller, the value of the goods is the price minus the T $\Delta \mathrm{S}$. Price is the equilibrium point of distribution value between buyer and seller, which is influenced by market supply and 
demand and various expectations.

For example: The value of a mobile phone is neither the cost nor the selling price of the phone, but the communication convenience and efficiency brought by the mobile phone to the customer, which is the difference between the expected value increase and the cost of the product. The value of the mobile phone is $\Delta \mathrm{H}$ minus the purchase price for the buyer; for the seller, the value of the mobile phone is the selling price minus $\mathrm{T} \Delta \mathrm{S}$ production cost. Trading is a process in which buyers and sellers share the value of goods and services at a price-cut point.

From the point of view of the Earth's ecosystem, the purpose of all human economic activities is to obtain, obtain efficiently and obtain more energy needed for survival and development, and obtain freedom to satisfy all kinds of desires. Wealth is the free power. It unifies the nature of freedom [9, 15-17] in literature, philosophy, politics, economics, ecology and physics---freedom is wealth, that is, free energy.

A definition of wealth $\mathrm{G}$ may be expressed as:

$$
\mathrm{G}=\mathrm{H}-\mathrm{TS}
$$

The $\mathrm{H}$ is the energy obtained by labor, TS is the energy consumed by labor.

1) $G$ represents the free energy of the system in physics, and in formula (2) it represents wealth. The essence of wealth is a form of freedom in human society. wealth is the accumulation of value $(\mathrm{G}=\Sigma \Delta \mathrm{G})$, representing an external ability to do work and empower, including, but not limited to, the storable energy, the energy of product solidification, the power of agreement to acquire energy (including real rights, claims, equity, futures, options, etc.).

If $\mathrm{H}-\mathrm{TS}<0$, means that the energy obtained by labor is less than the energy consumed by labor, it does not create wealth and value. In addition to humans, robots can also create wealth, and when they work more energy than they consume, they are also creating wealth and value.

Suppose a farmer eats $150 \mathrm{~kg}$ of wheat a year, but he only harvests $100 \mathrm{~kg}$ of wheat a year, so that instead of creating wealth, he loses $50 \mathrm{~kg}$ of wheat.

What needs to be explained is: early (fishing and hunting life period) mankind has intuitively realized that wealth comes from the gift of nature. For example, one raised cow and one wild cow have the same food value. At this time, the value is only related to the energy not to artificial breeding, and nature is also a wealth creator.

Robot farming on unmanned farms can produce $25000 \mathrm{~kg}$ of grain a year, deducting its rent and energy consumption for one year, and the rest is the wealth it creates. It can be seen that the theory of labor value is irrational in some aspects and has historical cognitive defects.

2) $\mathrm{H}$ represents the enthalpy of the system in physics, and in the formula (2) it represents the energy obtained by human beings through the process of purposeful activity (social labor).

This shows that labor is an activity of purposeful energy acquisition, and the purpose of labor is to obtain energy from the outside world and increase the enthalpy of the system. But labor does not necessarily create wealth, If labor can not acquire energy, it is doing nothing and not creating wealth.

3 ) in physics TS represents the energy consumed by the system to maintain its state, order, and structure. in formula (2) it represents the energy consumed by labor (social labor), that is, the energy consumed by human beings in the process of acquiring energy (activity, labor). It is manifested in the energy consumed by maintaining the ability to work under a certain quality of life (the value of the means of livelihood), Including the maintenance of lifestyle, living standards, social order and labor capacity of the total consumption of life (clothing, food, housing, travel, education, entertainment, etc.).

Because human and human society are dissipative structures, whether or not they work, maintaining their state, order and structure requires the consumption of energy to maintain basic metabolism, and failure to obtain basic survival energy will collapse and die. It should be explained that basic metabolic energy consumption is expressed as the cost of time in economics and is related to interest.

4) T represent the temperature of the system in physics, and in formula (2) it represents the quality of life, that is, the way of life and the standard of living, the degree of satisfaction of desire and the intensity of energy consumption.

5) S represents the entropy of the system in physics, and in formula (2) it represents the social order under a certain state and structure.

Because human society is a dissipative structure, it needs to consume energy continuously to produce negative entropy flow to maintain its state, order and structure.

The essence of order is consensus and coordination, and the state, order and structure of creating value can be guaranteed more efficiently through division of labor and cooperation. The humanities, art, science and so on can be regarded as to meet the needs of social division of labor and synergy. Its value is embodied in the coordination of social production and consumption activities through emotional synergy (joy, anger and sadness), consciousness synergy (thought and theory) and organizational synergy (behavior and goal), which is in line with historical culture and traditional habits.

\section{Energy Coins Are the Ideal Benchmark for Measuring Wealth and Value}

The earliest form of monetary value was to use some kind of goods with good circulation as general equivalents. Historically, goods such as shells, grains, livestock, tea, silk, fur, copper, iron, gold, silver and so on were used as general equivalents. All valuable things can act as general equivalents. With the expansion of trade scope, gold as a general equivalent becomes the world's common currency.

After 1972, the dollar was decoupled from gold, and with the joint efforts of governments, gold was exiled as an ordinary commodity on the precious metal exchange and gradually withdrew from the function of world currency. With sovereign credit as the endorsement of paper money, central 
banks abuse credit to issue paper money, create more than 40 years of sustained inflation, into the 21 st century after the world economy into a downturn.

Governments and central banks around the world want to promote economic growth by constantly issuing banknotes and intervening in markets, resulting in long-term inflation, diluting the wealth of the masses, widening the gap between the rich and the poor, leading to market failures, policy failures, and so on. Contradictions and problems accumulating, which seriously undermine the fairness and market order of human society. Paper money can not restrain the greed and laziness of the government, only the competition of various currencies can effectively restrict the spread of paper money, which is conducive to the prosperity and development of the world.

With the development of the Internet, digital payment gradually popularized, paper money gradually reduced circulation. Legal currency transactions have become the value in the bank database, and their circulation is no longer restricted by the production capacity of the Mint. Such a digital currency may be maliciously tampered with or cause all property to disappear instantly. In theory, methods such as disaster preparedness and internal control system can not eliminate the system risk and moral hazard inherent in this centralized structure. Internet digital economy era needs to restore non-main right currency as the world's common currency.

The digital currency [4] based on free energy is a monetary system in which the free energy state function $(\mathrm{G}=\mathrm{H}-\mathrm{TS})$ of physics is used to accurately calculate the free energy values contained in all goods and services. As a measure of value. This not only facilitates the unified measurement of the prices of various commodities, but also truly reflects the value of commodities. At the same time, it can automatically control the size of money according to the scale of the market of goods and services, and automatically prevent deflation and inflation. Commodity production and service providers, namely digital currency issuers, the value and scope of use of money are entirely determined by the market. The issuance of digital currency is cheap, easy to exchange, and can also circulate with many currencies at the same time. The customer and the seller have the right to decide in what currency to pay.

Energy coins are based on the energy of countless goods and services, is a physical decentralization, Free energy is measurable and easy to calculate, so energy coin is a natural decentralized digital currency that can reflect real value and can be used as a benchmark currency for measuring the value of various tokens.

\section{Relationship Between Currencies}

In human history, gold and silver have been used as the world's common currency, which is created by gold prospectors and value creators. This currency itself is an equivalent and does not need credit at all. Paper money requires credit, which is why governments and central banks issue paper money. After that, the IMF used a "basket" of legal tender as the world currency. Bitcoin is not only the innovation of digital currency mint technology, but also an innovation of currency issuing system and mechanism. Nakamoto created a truly decentralized self-organized digital money ecosystem with this principle open, source code open, and no issuing and managing subjects. Decentralization refers to the return of the right to issue money from the central bank to the value producer. One of the core values of Bitcoin is the concept of decentralization of coinage, which makes it possible to return coinage rights to value creators.

If not decentralized, digital currency does not need block chain technology, it is only legal currency digitization. The purpose of bitcoin is to reduce the high intermediary credit cost and risk by decentralization. Although the design idea of bitcoin [5] is classic, it has four design defects that make it unable to assume the role of the world's universal currency:

1) the same scarcity as gold will inevitably lead to deflation;

2) there is no value base support, history has proved that consensus is unreliable, must have value base support;

3) Bitcoin mining costs are too high;

4) not fully consider AI application scenarios in the field of digital money.

Bitcoin is more suitable for storing large wealth, secret transactions, large exchange and other scenarios.

Nakamoto is the founder of decentralized digital money technology and the pioneer of block chain technology. Energy coin is the value chain which is constructed and superimposed on the block chain, the perfection and development of Bitcoin, and the value chain and value transmission protocol built by the free energy of goods and services. The core of value is energy and consensus, which builds consensus and values based on algorithms such as ecology, politics, economics, physics, mathematics, cryptography and so on. Energy coins realize the securitization of the complete value chain.

The basic technical framework of energy coin is digital cash based on Internet, free energy as value chain algorithm and bitcoin block chain technology. The relevant knowledge structures of the Internet, blockchain and value chains are described in Table 2.

Table 2. Internet, Block chain and Value chain.

\begin{tabular}{ll}
\hline Ecology Politics & energy coin \\
Economics Physics & value chain \\
Mathematics Cryptography & Bitcoin \\
Programming Software engineering & Block chain \\
Communications agreements & Internet TCP / IP \\
Communications engineering & Telecommunications Network \\
\hline
\end{tabular}

\section{Energy Coins are the Ideal World Currency}

Digital currency is the inevitable product of the Internet digital age. Energy coin can become the medium of intelligent contract automatic deal-making value chain algorithm. Internet, AI and digital technology will gradually network, flatten and decentralize the social structure, and make natural persons, enterprises, governments and various social groups become equal market subjects. 
Energy coin is a value chain digital currency based on block chain technology. It is a monetary system that can automatically control the size of money according to the scale of goods and services market. It solves the pain points of various currencies in history, such as gold deflation, inflation of legal currency, and the malpractice of no value base of digital currency such as Bitcoin. Energy coin is a rational consensus based on natural laws such as physical laws and mathematical algorithms based on energy as a general equivalent. It is the most ideal universal currency in the world. Energy coin is a kind of non-state super-sovereign that is not controlled by any individual, organization or state.

Energy and time, space, mass and other physical quantities are objective and measurable, Energy coin is the basic physical quantity free energy as the carrier of the digital coin. Like gold, it can carry the exchange, circulation, storage and inheritance of value and wealth through time and space, and can guarantee the security of personal wealth through the rise and fall of the country, the change of regime, the change of race, the religious belief and so on. Energy coins as the basic characteristics of the world currency:

1) must be truly decentralized, without specific beneficiaries, without specific managers, without the need for specific people or organizations to operate and maintain. Developers, like Nakamoto, must decentralize themselves.

2) must be principle open, source code open, no person control, self-organization, automatic operation.

3) can not be regulated and shut down by any government, organization or individual, nor controlled or shut down by the founder

4) energy coins should be issued by value creators, gold miners and miners issuing world currency as gold did in the year.

5) like Bitcoin, does not require the approval, licensing and regulation of any government, organization and institution, and relies entirely on consensus and market to achieve issuance, trading and circulation.

6) can be separated from the banking system in the market free circulation, storage. The owner is free to control, like gold.

7) must be non-nationalized, super-sovereign and do not require credit endorsement by any organization.

8) energy coins represent the freedom, value and wealth of the future human world

\section{Conclusion}

1) Energy coin is a decentralized digital currency based on free energy and block chain technology.

2) Energy coins are based on the energy contained in countless goods and services, are physically decentralized, and free energy is measurable and computational, so energy coins are an ideal decentralized super-sovereign currency that is not manipulated by any individual, organization or state, and unifies the self-organization mechanism of the earth's ecosystem with the market self-organization mechanism. Energy coin is a qualitative leap in the history of money and an ideal universal currency in the world.

3) Energy coin can be used as the universal digital currency in the future digital world. it solves the drawbacks of various currencies in the past, such as the deflation of gold, the inflation of legal currency, the worthless basis of digital currency such as Bitcoin, etc. It is the ideal universal currency to construct a more fair, efficient and global new world economic order.

4) Energy coin touches the core interests of human society and the most important monetary problems. It inevitably touches the nerves of politics, economy, law, philosophy, values and so on. It is far from a simple technical problem. The essence of value is the free energy created by producers, and the issue of money by value creators should become a global consensus. Decentralization of the non-national monetary era is the Internet digital society of human progress inherent requirements.

\section{References}

[1] Kang Jian, "Macrodynamic of Earth Ecosystem", J. Solar Energy, 2013, (3): pp. 8-12.

[2] Kang Jian, "General Theory of Life, Energy and Value Measurement (I)” J. Solar Energy, 2014, (6): pp. 24-27.

[3] Kang Jian, "General Theory of Life, Energy and Value Measurement (II)”, J. Solar Energy, 2014, (7): pp. 10-13.

[4] Kang Jian, "A universal digital currency based on free energy" P. China: 20181019411764.

[5] Satoshi Nakamoto. "Bitcoin: A Peer-to-Peer Electronic Cash System" EB/OL. https://bitcoin.org/bitcoin.pdf.

[6] Isaac Newton, "Mathematical Principles of Nature Philosophy" ISBN: 9787100045131, The Commercial Press 2006-7-1 Translator: Zhao Zhenjiang.

[7] Miao Qilong, "An Outline of Earth Sciences" ISBN: 9787502931308, China Meteorological Press 2007-8-1.

[8] Adam Smith, "An Inquiry into the Nature and Causes of the Wealth of Nations" ISBN: 9787504930279, China Finance Press 2003-6, Translator: Guo Dali, Wang Yanan.

[9] Milton Friedman, "Capitalism and Freedom", ISBN: 9787100041676, The Commercial Press 2004-7, Translator: Zhang Ruiyu.

[10] John Maynard Keynes, "The General Theory of Employment, Interest and Money", ISBN: 9787100026147 , The Commercial Press 1999-4, Translator: Gao Hongye.

[11] John Maynard Keynes, “A Treatise on Money", ISBN: 9787212055769, Anhui peoples Publishing House 2012-10, Translator: Deng Chuanjun, Liu Zhi.

[12] Cheng Jianchun, "Introduction to Theoretical Physics" ISBN: 9787030185549, Science Press 2007-04-01. 
[13] Ilya Prigogine, "Self-Organization in Non-Equilibrium Systems" BN: 13031-3154, Science Press 1986-5, Translator: $\mathrm{Xu}$ Xishen, Chenigang, Wang Guangrui, Chen Ya.

[14] Ilya Prigogine, "Introduction to Thermodynamics of Irreversible Processes” BN: 13031-1418, Science Press 1960-8, Translator: Xu Xi.

[15] Friedrich August von Hayek, "The Constitution of Liberty", ISBN: 9787108011046, Sanlian Bookstore Press 1997-12, Translator: Deng Zhenglai.
[16] Friedrich August von Hayek, "The Road to Serfdom", ISBN: 9787500421368, China social sciences press 1997-08-01, Translator: Wang Mingyi, Feng Xing.

[17] Aristotle, "Politics" ISBN: 9787100018036, China Renmin University Press 1965-8-1, Translator: Wu Shoupeng.

[18] Eugene P. Odum, "Fundament of Ecology", ISBN: 9787040251531, Higher Education Press 2009-1, Translator: Lu Jianjian, Wang Wei, Wang Tianhui, Ho Wenshan, Li Xiuzhen. 